Brasil, J, Pereira, LM, Pistori, MES, Mezzomo. TR \& Zaparolli, MR. (2020). Quality assessment of enteral nutritional therapy in an intensive care unit. Research, Society and Development, 9(7):1-13, e218973685.

\title{
Avaliação da qualidade da terapia nutricional enteral em um serviço de terapia
} intensiva

Quality assessment of enteral nutritional therapy in an intensive care unit Evaluación de la calidad de la terapia nutricional enteral en una unidad de cuidados intensivos

Recebido: 15/04/2020 | Revisado: 15/04/2020 | Aceito: 04/05/2020 | Publicado: 07/05/2020

Jessica Brasil

ORCID: https://orcid.org/0000-0003-3513-276X

Universidade Positivo, Brasil E-mail: jessicabrasil.lora@gmail.com

Larissa Marques Pereira ORCID: https://orcid.org/0000-0002-1294-8822 Universidade Positivo, Brasil E-mail: larissatoninmarques@gmail.com Mary Evelyn Sbalqueiro Pistori Hospital do Trabalhador, Brasil ORCID: https://orcid.org/0000-0001-5249-1161 E-mail: marypistori@sesa.pr.gov.br Thais Regina Mezzomo ORCID: https://orcid.org/0000-0002-0884-8052 Centro Universitário Internacional Uninter, Brasil E-mail: thaismezzomo@yahoo.com.br Marília Rizzon Zaparolli ORCID: https://orcid.org/0000-0002-5112-6979 Universidade Positivo, Brasil E-mail: mariliazaparolli@gmail.com

\section{Resumo}

O objetivo do estudo foi analisar os indicadores de qualidade em terapia nutricional enteral em pacientes admitidos em uma unidade de terapia intensiva (UTI) de um hospital de 
referência em trauma. Para tanto, um estudo observacional retrospectivo foi realizado com pacientes com idade $\geq 18$ anos, que receberam terapia nutricional enteral exclusiva por pelo menos 72 horas na UTI, no período de maio a setembro de 2017. Foram coletados dados demográficos, diagnóstico médico, tempo de início da infusão da nutrição enteral, informações sobre a nutrição prescrita versus infundida, número de pacientes que atingiram $50 \%, 70 \%$ e $100 \%$ de energia e proteína em três, cinco e sete dias respectivamente, além de sintomas gastrointestinais presentes durante a terapia nutricional enteral. Foram elegíveis dados de 96 pacientes. Observou-se que $77 \%$ da amostra estava internada devido à trauma e $11,34 \%$ apresentaram frequência de jejum maior que 48 horas. A adequação do teor de nutrição enteral infundido versus prescrito foi de $83,42 \%$. Apenas $44,8 \%$ dos pacientes atingiram 50\% da energia recomendada em 3 dias, enquanto que 57,2\% atingiram $70 \%$ em 5 dias e 44,2\% atingiram 100\% em 7 dias. Quanto a ingestão proteica, 46,9\% atingiram 50\% da ingestão recomendada em 3 dias; 57,2 \% atingiram 70\% em 5 dias e 41,5\% atingiram 100\% em 7 dias. A frequência de sintomas gastrointestinais foi de 44,7\%. Aproximadamente metade da amostra não alcançou progressivamente os percentuais de energia e proteínas desejados. Recomenda-se monitorar a evolução nutricional, identificando as situações e estabelecer intervenções para a progressão nutricional do paciente.

Palavras-chave: Terapia nutricional; Unidade de terapia intensiva; Indicadores de qualidade em assistência à saúde.

\section{Abstract}

The aim of the study was to analyze the quality indicators in enteral nutritional therapy in patients admitted to an intensive care unit (ICU) of a trauma reference hospital. For this purpose, a retrospective observational study was carried out with patients aged $\geq 18$ years, who received exclusive enteral nutritional therapy for at least 72 hours in the ICU, from May to September 2017. Demographic data, medical diagnosis, time to start infusion of enteral nutrition, information on prescribed versus infused nutrition, number of patients reaching $50 \%, 70 \%$ and $100 \%$ of energy and protein in three, five and seven days, respectively, were collected, in addition to gastrointestinal symptoms present during enteral nutritional therapy. Data from 96 patients were eligible. It was observed that $77 \%$ the sample was hospitalized due to trauma and $11.34 \%$ had a fasting frequency greater than 48 hours. The adequacy of the volume of infused versus prescribed nutrition was $83.42 \%$. Only $44.8 \%$ of patients reached $50 \%$ of the recommended energy in 3 days, while $57.2 \%$ reached $70 \%$ in 5 days and 44.2 reached $100 \%$ in 7 days. As for protein intake, $46.9 \%$ reached $50 \%$ of the recommended 
intake within 3 days; $57.2 \%$ reached $70 \%$ in 5 days and $41.5 \%$ reached $100 \%$ in 7 days. The frequency of gastrointestinal symptoms was $44.7 \%$. Approximately half of the sample did not progressively reach the desired energy and proteins percentages. It is recommended to monitor the nutritional evolution, identifying the problems and establishing interventions for the patient's nutritional progression.

Keywords: Nutritional therapy; intensive care unit; quality indicators in healthcare.

\section{Resumen}

El objetivo del estudio fue analizar los indicadores de calidad en la terapia nutricional enteral en pacientes ingresados en una unidad de cuidados intensivos (UCI) de un hospital de referencia de trauma. Para ello, se realizó un estudio observacional retrospectivo con pacientes de $\geq 18$ años que recibieron terapia nutricional enteral exclusiva por lo menos 72 horas em la UCI, de mayo a septiembre de 2017. Se recopilaron datos demográficos, diagnóstico médico, tiempo de inicio de la infusión de nutrición enteral, información sobre nutrición prescrita versus infundida, número de pacientes que alcanzaron el 50\%, 70\% y $100 \%$ de energía y proteínas en tres, cinco y siete días respectivamente, además de los síntomas gastrointestinales presentes durante terapia nutricional enteral. Los datos de 96 pacientes fueron elegibles. Se observó que el $77 \%$ de la muestra fue hospitalizada debido a un traumatismo y el 11,34\% tenía una frecuencia de ayuno mayor de 48 horas. La adecuación del volumen de infusión versus nutrición prescrita fue del $83,42 \%$. Solo el $44,8 \%$ de los pacientes alcanzaron el 50\% de la energía recomendada en 3 días, mientras que el 57,2\% alcanzó el $70 \%$ en 5 días y 44,2\% alcanzó el 100\% en 7 días. En cuanto a la ingesta de proteínas, el 46,9\% alcanzó el 50\% de la ingesta recomendada en 3 días; el 57,2\% alcanzó el $70 \%$ en 5 días y el 41,5\% alcanzó el 100\% en 7 días. La frecuencia de síntomas gastrointestinales fue del $44,7 \%$. Aproximadamente la mitad de la muestra no alcanzó progresivamente los porcentajes deseados de energía y proteína. Se recomienda controlar la evolución nutricional, identificar los problemas y establecer intervenciones para la progresión nutricional del paciente.

Palabras clave: Terapia nutricional; Unidad de Terapia Intensiva; Indicadores de calidad en asistencia a la salud. 


\section{Introdução}

No âmbito hospitalar, a desnutrição gera preocupações, pois está correlacionada com a piora da resposta imunológica assim como comprometimento no reparo tecidual, acarretando aumento do risco de infecção, tempo de internação, custo à saúde pública e maior índice de mortalidade (Couto, Moreira \& Hoher, 2012; Oliveira Filho, 2016). Em unidades de terapia intensiva (UTI) a desnutrição varia de 38 a $83 \%$ dos hospitalizados e tende a aumentar até o momento da alta hospitalar (Hejazi et al., 2016; Kimiaei-Asadi \& Tavakolitalab, 2017; Lec et al., 2017).

A terapia nutricional enteral (TNE) é uma alternativa terapêutica para os pacientes que não conseguem atingir suas necessidades nutricionais espontaneamente, sendo recomendado seu início precoce entre 24 a 48 horas após admissão hospitalar, atingindo 50 a $70 \%$ das necessidades energéticas e proteicas ainda na fase inicial da internação (Kim et al., 2013; Mcclave et al., 2016; Paese, Nascimento \& Nascimento, 2016; Gomes, Cabral \& Oliveira, 2017; Castro et al., 2018; Kampa et al., 2020).

O acompanhamento nutricional no paciente crítico é de extrema importância para garantir a adequada oferta nutricional, auxiliar na evolução clínica favorável e promover a qualidade de vida do paciente (Castro et al., 2018). Para tanto, a avaliação e o acompanhamento nutricional envolvem parâmetros clínicos, antropométricos, bioquímicos e dietéticos, os quais, devido as alterações metabólicas decorrentes do quadro clínico do paciente, dificultam a obtenção e o monitoramento desses métodos (Mcclave et al., 2016; Castro et al., 2018; Lesniovski et al., 2018). Além disso, intercorrências gastrointestinais como êmese, distensão abdominal, estase gástrica, diarreia e tempo prolongado de jejum podem interferir na oferta de nutrientes, na cicatrização, na evolução clínica e no estado nutricional do paciente (Souza \& Mezzomo, 2016; Lesniovski et al., 2018; Sampaio et al., 2019). Assim, preconiza-se a utilização de indicadores de qualidade na assistência nutricional, ferramenta útil para nortear e avaliar a qualidade do cuidado ao paciente e para identificar falhas e dificuldades no fluxo de atendimento em uma UTI (Waitzberg, 2010; Passinato et al, 2013; Souza \& Mezzomo, 2016; Lesniovski et al., 2018; Sampaio et al., 2019). Considerando que a inadequação de nutrientes pode acarretar comprometimento do estado nutricional e aumento da morbimortalidade, o objetivo deste estudo foi avaliar indicadores de qualidade em terapia nutricional de pacientes sob TNE admitidos em uma UTI de um hospital público de referência em trauma.

\section{Metodologia}

Trata-se de uma pesquisa de campo quantitativa, observacional descritiva, de temporalidade retrospectiva (Pereira et al., 2018), realizada a partir da análise de dados de fichas de acompanhamento nutricional de pacientes hospitalizados em uma UTI de um hospital público, com 
referência em trauma, na cidade de Curitiba, Paraná, Brasil. O estudo foi aprovado pelo Comitê de Ética em Pesquisa da Universidade Positivo sob o nº 2.714.210.

Os critérios de inclusão de pacientes no estudo foram pacientes com idade maior ou igual a18 anos, internados na UTI e que receberam TNE exclusiva por pelo menos 72 horas, com internamento no período de maio a setembro de 2017. Foram coletados dados referentes a idade, sexo, diagnóstico médico, tempo em jejum antes do início da infusão da nutrição enteral ( $\leq 48$ horas e $>48$ horas), volume de nutrição enteral prescrita versus infundida, número de pacientes que atingiram $50 \%, 70 \%$ e $100 \%$ de energia e proteína em três, cinco e sete dias respectivamente, além da frequência de pacientes que apresentaram eventos do trato gastrointestinal (TGI) como êmese, estase gástrica e diarreia. Os indicadores de qualidade em terapia nutricional foram avaliados e classificados conforme o Quadro 1 (Waitzberg, 2010; Pasinato et al., 2013).

Quadro 1. Indicadores e metas de qualidade em terapia nutricional enteral.

\begin{tabular}{|c|c|c|}
\hline $\begin{array}{c}\text { Indicadores de qualidade em } \\
\text { terapia nutricional }\end{array}$ & Fórmulas & Metas \\
\hline $\begin{array}{l}\text { (1) Frequência de pacientes em } \\
\text { jejum antes do início da TNE }\end{array}$ & $\begin{array}{l}\mathrm{N}^{\circ} \text { de pacientes candidatos a TNE em jejum }>48 \mathrm{~h} \times 100 / \\
\mathrm{n}^{\circ} \text { total de candidatos a TNE }\end{array}$ & $<20 \%$ \\
\hline $\begin{array}{l}\text { (2) Taxa de adequação do } \\
\text { volume infundido em relação ao } \\
\text { prescrito em pacientes em TNE }\end{array}$ & $\begin{array}{c}\text { Volume total de dieta infundida no mês x } 100 / \\
\text { volume total de dieta prescrita no mês }\end{array}$ & $\geq 80 \%$ \\
\hline $\begin{array}{l}\text { (3) Frequência de } \\
\text { intercorrências relacionadas ao } \\
\text { TGI }\end{array}$ & $\begin{array}{l}\mathrm{N}^{\circ} \text { de pacientes com intercorrências relacionadas ao TGI x } 100 \\
\qquad / \mathrm{n}^{\circ} \text { total de pacientes em TNE }\end{array}$ & $<5 \%$ \\
\hline $\begin{array}{l}\text { (4) Porcentagem de pacientes } \\
\text { que atingiram } 50 \% \text { das } \\
\text { necessidades energéticas em } 3 \\
\text { dias }\end{array}$ & $\begin{array}{l}\mathrm{N}^{\circ} \text { de pacientes que atingiram } 50 \% \text { das necessidades } \\
\text { energéticas em } 3 \text { dias } \times 100 / \mathrm{n}^{\circ} \text { total de pacientes em TNE }\end{array}$ & - \\
\hline $\begin{array}{l}\text { (5) Porcentagem de pacientes } \\
\text { que atingiram } 70 \% \text { das } \\
\text { necessidades energéticas em } 5 \\
\text { dias }\end{array}$ & $\begin{array}{l}\mathrm{N}^{\circ} \text { de pacientes que atingiram } 70 \% \text { das necessidades } \\
\text { energéticas em } 5 \text { dias } \times 100 / \mathrm{n}^{\circ} \text { total de pacientes em TNE }\end{array}$ & - \\
\hline $\begin{array}{l}\text { (6) Porcentagem de pacientes } \\
\text { que atingiram } 100 \% \text { das } \\
\text { necessidades energéticas em } 7 \\
\text { dias }\end{array}$ & $\begin{array}{l}\mathrm{N}^{\circ} \text { de pacientes que atingiram } 100 \% \text { das necessidades } \\
\text { energéticas em } 7 \text { dias x } 100 / \mathrm{n}^{\circ} \text { total de pacientes em TNE }\end{array}$ & - \\
\hline $\begin{array}{l}\text { (7) Porcentagem de pacientes } \\
\text { que atingiram } 50 \% \text { das } \\
\text { necessidades proteicas em } 3 \\
\text { dias }\end{array}$ & $\begin{array}{c}\mathrm{N}^{\circ} \text { de pacientes que atingiram } 50 \% \text { das necessidades proteicas } \\
\text { em } 3 \text { dias } x 100 / \mathrm{n}^{\circ} \text { total de pacientes em TNE }\end{array}$ & - \\
\hline $\begin{array}{l}\text { (8) Porcentagem de pacientes } \\
\text { que atingiram } 70 \% \text { das } \\
\text { necessidades proteicas em } 5 \\
\text { dias }\end{array}$ & $\begin{array}{c}\mathrm{N}^{\circ} \text { de pacientes que atingiram } 70 \% \text { das necessidades proteicas } \\
\text { em } 5 \text { dias } x 100 / \mathrm{n}^{\circ} \text { total de pacientes em TNE }\end{array}$ & - \\
\hline $\begin{array}{l}\text { (9) Porcentagem de pacientes } \\
\text { que atingiram } 100 \% \text { das } \\
\text { necessidades proteicas em } 7 \\
\text { dias }\end{array}$ & $\begin{array}{c}\mathrm{N}^{\circ} \text { de pacientes que atingiram } 100 \% \text { das necessidades proteicas } \\
\text { em } 7 \text { dias } \times 100 / \mathrm{n}^{\circ} \text { total de pacientes em TNE }\end{array}$ & - \\
\hline
\end{tabular}


Research, Society and Development, v. 9, n. 7, e218973685, 2020

(CC BY 4.0) | ISSN 2525-3409 | DOI: http://dx.doi.org/10.33448/rsd-v9i7.3685

O Quadro 1 fornece as equações utilizadas para a obtenção dos indicadores de qualidade, bem como, as metas para referência dos valores obtidos.

Os dados da pesquisa foram compilados e analisados no programa BioEstat $\AA$. Os dados quantitativos foram descritos por mensuração de tendência central e dispersão. Os dados qualitativos foram apresentados segundo suas frequências absolutas. $O$ teste quiquadrado, com nível de significância de $\mathrm{p}<0,05$, foi aplicado.

\section{Resultados}

Foram analisados 476 fichas de atendimento nutricional de pacientes internados na UTI no período de maio a setembro de 2017 e foram elegíveis dados de somente $20,3 \%$ (n = 96) dos pacientes, sendo $29 \%(\mathrm{n}=28)$ do sexo feminino e $71 \%(\mathrm{n}=68)$ do sexo masculino. A mediana da idade foi de 48 (18-97) anos. A descrição da população do estudo encontra-se na Tabela 1.

Tabela 1- Caracterização da população de pacientes em terapia nutricional enteral internados em uma unidade de terapia intensiva de um hospital de trauma.

\begin{tabular}{lcc}
\hline \multicolumn{1}{c}{ Característica } & $\%(\mathbf{n})$ & $\mathbf{p}$ \\
\hline Sexo & & $<0.0001$ \\
Feminino & $29 \%(\mathrm{n}=28)$ & \\
Masculino & $71 \%(\mathrm{n}=68)$ & \\
\hline Diagnóstico médico & $77 \%(\mathrm{n}=74)$ & \\
Trauma & $11,4 \%(\mathrm{n}=11)$ & \\
Infecção & $8 \%(\mathrm{n}=8)$ & $<0.0001$ \\
Evento cardiológico & $2 \%(\mathrm{n}=2)$ & \\
Evento neurológico & $1 \%(\mathrm{n}=1)$ & \\
Intoxicação química & $12(4-94)$ & \\
\hline Tempo de internação na UTI & & \\
(dias) & & \\
\hline Nota: UTI: unidade de terapia intensiva. & & \\
Teste qui-quadrado, $\mathrm{p}<0,05$. &
\end{tabular}

Em relação ao indicador de qualidade em TNE (1), verificou-se que 88,5\% (n=85) dos pacientes iniciaram TNE nas primeiras 48 horas de internação e 11,5\% (n=11) após 48 horas ( $\mathrm{p}<0.001)$, resultado esse que apresentou adequação à meta estipulada de $<20 \%$ dos pacientes em jejum prolongado. Quanto ao indicador de qualidade (2), a taxa de adequação do 
volume de nutrição enteral infundido versus prescrito foi de $83,4 \%$, resultado esse que cumpriu a meta preconizada de $\geq 80 \%$.

Sobre o indicador (3) frequência de intercorrências relacionadas ao TGI, observou-se que $44,7 \%(n=44)$ dos pacientes analisados apresentaram êmese, diarreia e estase gástrica. Dentre estes sintomas, a diarreia foi a complicação mais frequente (Figura 1).

Figura 1. Intercorrências relacionadas ao trato gastrointestinal em pacientes sob terapia nutricional enteral internados em uma unidade de terapia intensiva de um hospital de trauma.

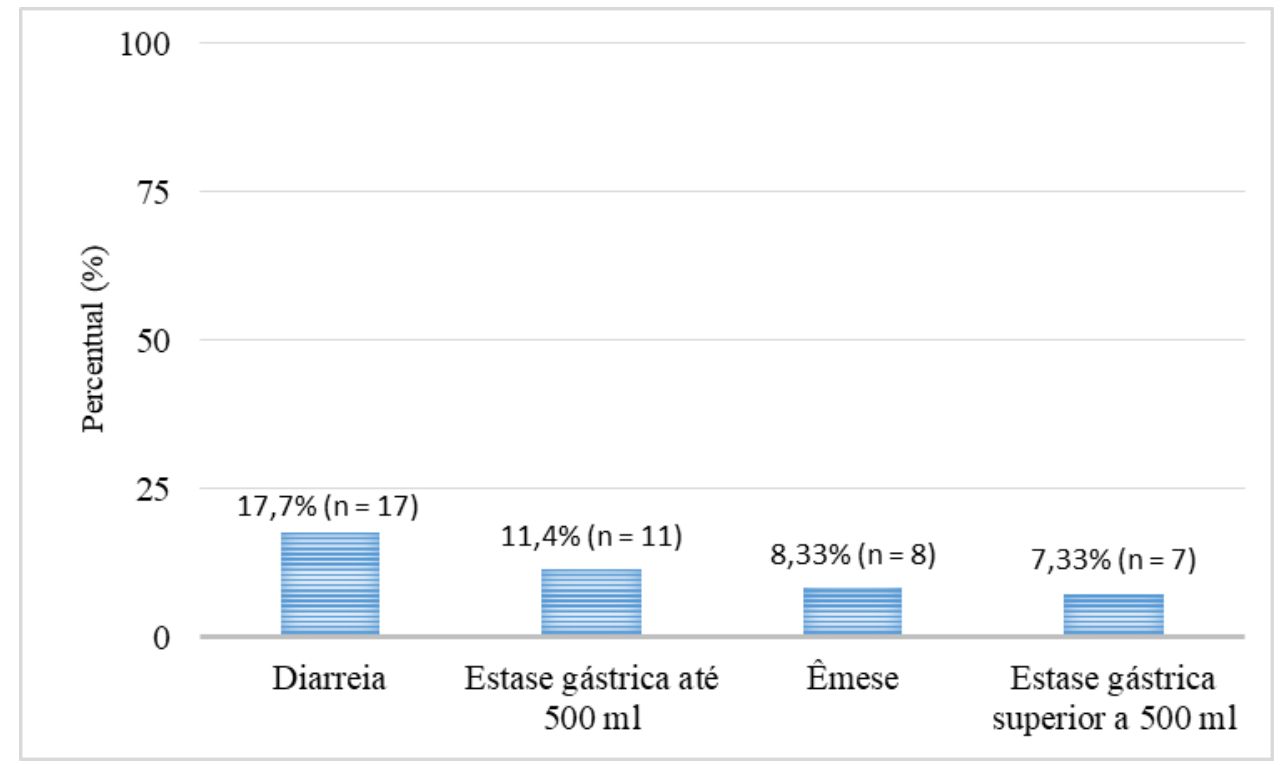

Conforme observado na Figura 1, o indicador de qualidade em TNE relacionado às intercorrências do trato gastrointestinal não apresentou adequação à meta estipulada de 5\%, visto que os sintomas variaram entre $7,33 \%$ a $17,7 \%$ de frequência.

Os resultados dos indicadores de qualidade (4 a 9) sobre as metas de proteína e energia atingidas pelos pacientes podem ser visualizados na Tabela 2. Não houve diferença estatística significativa entre os grupos. 
Tabela 2 - Metas de ingestão proteica e energética em relação ao tempo de internação de pacientes em terapia nutricional enteral internados em uma unidade de terapia intensiva de um hospital de trauma.

\begin{tabular}{lccc}
\hline Metas & $\begin{array}{c}\text { Atingiram a meta } \\
\text { estabelecida (\%) }\end{array}$ & $\begin{array}{c}\text { Não atingiram a meta } \\
\text { estabelecida (\%) }\end{array}$ & p \\
\hline Proteína & & & \\
$50 \%(3$ dias) & $46,9 \%(\mathrm{n}=45)$ & $53,1 \%(\mathrm{n}=51)$ & 0,540 \\
$70 \%(5$ dias) & $57,2 \%(\mathrm{n}=52)$ & $58,8 \%(\mathrm{n}=39)$ & 0,149 \\
$100 \%(7$ dias $)$ & $41,5 \%(\mathrm{n}=32)$ & & 0,090 \\
\hline Energia & & $55,2 \%(\mathrm{n}=45)$ & 0,298 \\
$50 \%(3$ dias $)$ & $44,8 \%(\mathrm{n}=43)$ & $42,8 \%(\mathrm{n}=39)$ & 0,152 \\
$70 \%(5$ dias) & $57,2 \%(\mathrm{n}=52)$ & $55,8 \%(\mathrm{n}=43)$ & 0,246 \\
$100 \%(7$ dias $)$ & $44,2 \%(\mathrm{n}=34)$ & & \\
\hline Nota: \%: percentual. & & & \\
Teste qui-quadrado, $\mathrm{p}<0,05$. & & &
\end{tabular}

Conforme a Tabela 2, aproximadamente metade da amostra alcançou a meta estipulada de proteínas e energia, conforme os dias de evolução de terapia nutricional. Em especial, após 7 dias, $58,4 \%$ não atingiram a meta estipulada para proteína e 55,8\% não atingiram a recomendação de energia.

\section{Discussão}

A TNE faz parte da atenção nutricional de indivíduos em cuidados intensivos e o iníco precoce visa manutenção funcional do TGI e adequada nutrição (Mcclave et al., 2016; Castro et al., 2018). Quando iniciada precocemente, A TNE está relacionada a menor risco de complicações clínicas, de mortalidade e menor tempo de internação (Chourdakis et al., 2010). Neste estudo a maioria dos pacientes teve TNE iniciada precocemente, em até $48 \mathrm{~h}$ da internação, resultado esse de acordo com as diretrizes nacionais e internacionais de nutrição em terapia intensiva (Mcclave et al., 2016; Castro et al., 2018). Segundo a literatura, os motivos frequentes que culminam em início tardio de TNE em UTI são a instabilidade hemodinâmica e o jejum para exames e cirurgias, situações essas comuns em UTI com pacientes politraumatizados (Paese, Nascimento \& Nascimento, 2016). Ressalta-se que nas fichas de acompanhamento nutricional avaliadas não estavam descritos os motivos do atraso do início da alimentação enteral, apenas a data do seu início, portanto, não foi possível analisar as possíveis causas. 
O indicador de qualidade (2), referente as taxas de volume de nutrição enteral prescrito versus infundido mostrou adequação à meta estabelecida pelas diretrizes nacionais e internacionais (Waitzberg, 2010; Mcclave et al., 2016; Castro et al., 2018). Este resultado corrobora com resultados previamentos descritos nesta UTI em anos anteriores (Lesniovski et al., 2018). Entretanto, este resultado difere de outros estudos (Santana, Vieira, Dias, Braga \& Costa, 2016; Menezes, Silva, Cutunda, de Gois \& Oliveira, 2018). A adequação da quantidade de nutrição prescrita contribui para a adequação ou manutenção do estado nutricional do paciente, cicatrização de feridas, minimiza tempo de internamento, otimiza custos e diminui complicações (Pasinato et al., 2013). O paciente crítico necessita de maior atenção e controle em protocolos de cuidado em TNE e, portanto, deve haver aprimoramento constante da EMTN (Sampaio et al., 2019).

Quanto ao indicador de qualidade (3) sobre intercorrências relacionadas ao TGI, os resultados aqui encontrados são elevados e não adequados à meta proposta de 5\%, contudo, são semelhantes a outros estudos (Souza \& Mezzomo, 2016; Lesniovski et al., 2018). A diarreia é a complicação gastrointestinal mais comum e afeta a dignidade do paciente, aumenta a carga de trabalho do serviço de enfermagem e os custos em saúde, exacerba a morbidade por meio de lesão dérmica, prejudica a absorção de nutrientes, causa subsequente desequilíbrio hidroeletrolítico, aumenta o tempo de internamento da UTI e a mortalidade (Tirlapur et al., 2016), bem como, o quadro diarreico é associado à menor frequência de administração adequada de energia e do volume de nutrição enteral infundido quando comparado ao prescrito (Sampaio et al., 2019). Os principais fatores associados à ocorrência de diarreia são febre, uso de laxantes e/ou enemas, hipoalbuminemia, uso de antibióticos da classe dos carbapenêmicos e glicopeptídeos, além de medicamentos procinéticos (Rodrigues et al, 2018). Por outro lado, a êmese e a estase gástrica podem ocasionar aspiração brônquica e evoluir para pneumonia aspirativa, que interfere negativamente na evolução do paciente (Souza \& Mezzomo, 2016).

No presente estudo, menos da metade dos pacientes atingiram a meta de 50 e $100 \%$ das necessidades energéticas e proteicas nos primeiros três e sete dias, respectivamente. Resultados da literatura apresentam resultados similares (Passinato et al., 2013). Contudo, no sétimo dia de TNE, esse trabalho verificou que mais da metade dos pacientes apresentaram adequação de 70\%. Esse resultado é levemente superior a outros estudos (Passinato et al., 2013). O principal motivo dos pacientes não atingirem as metas energéticas e proteicas é a interrupção da administração da nutrição enteral devido a procedimentos (Passinato et al., 2013; Mcclave et al., 2016). Dessa forma, que é preciso monitorar e adequar a prescrição e a 
(CC BY 4.0) | ISSN 2525-3409 | DOI: http://dx.doi.org/10.33448/rsd-v9i7.3685

administração da nutrição enteral para o estado hipercatabólico em razão da resposta inflamatória exacerbada resultante da injúria, reparo tecidual e fornecimento de substratos ao sistema imunológico, tornando a necessidade proteica primordial e maior. A oferta adequada de proteína é necessária para a adequada cicatrização de feridas, manutenção da função imunológica e da massa magra (Passinato et al., 2013; Mcclave et al., 2016). Considera-se comumente que o aumento da ingestão de energia e proteína em pacientes críticos diminui a mortalidade (Waitzberg, 2010).

Esse estudo ressalta a dificuldade em alimentar pacientes sob TNE em UTI. Contudo, apresenta como limitação a não avaliação das causas da interrupção e/ou da não administração adequada da TNE, visto a ausência de informações nas fichas de acompanhamento nutricional.

\section{Conclusão}

Esse estudo demonstrou que pacientes críticos em UTI recebem TNE precocemente com volume de nutrição enteral adequado conforme prescrição nutricional. Contudo, intercorrências relacionadas ao TGI são frequentes e podem ser um dos motivos que levam a não adequação de energia e proteína necessária para a maioria dos pacientes da amostra avaliada.

Sugere-se que novos trabalhos avaliem a evolução da infusão de nutrientes de acordo com a evolução do paciente na UTI e monitorem as causas de inadequação nutricional para estabelecer medidas de melhorias em pontos de não conformidade e melhorar a qualidade do serviço ofertado aos pacientes críticos.

\section{Referências}

Castro, M. G., Ribeiro, P. C., Souza, I. A. O., Cunha, H. F. R., Silva, M. H. N., Rocha, E. E. M., Correia, F. G., Loss, S. H., Franco Filho, J. W., Nunes, D. S. L., Gonçalves, R. C., Matos, L. B. N., Ceniccola, G. D. \& Toledo, D. O. (2018). Diretriz brasileira de terapia nutricional no paciente grave. Braspen J, 33(Supl 1): 2-36.

Chourdakis, M., Kraus, M. M., Tzellos, T., Sardeli, C., Peftoulidou, M., Vassilakos, D. \& Kouvelas, D. (2012). Effect of early compared with delayed enteral nutrition on endocrine 
function in patients with traumatic brain injury: an open-labeled randomized trial. J Parenter Enteral Nutr, 36(1):108-16.

Couto, C. F. L., Moreira, J. S. \& Hoher, J. A. (2012). Terapia nutricional enteral em politraumatizados sob ventilação mecânica e oferta energética. Rev Nutr, 25(6): 695-705.

Gomes, R. D. S., Cabral, N. A. L. \& Oliveira, A. T. V. (2017). Qualidade da terapia nutricional enteral em unidades de terapia intensiva. Braspen J, 32(2): 162-169.

Hejazi, N., Mazloom, Z., Zand, F., Rezaianzadeh, A. \& Amini, A. (2016). Nutritional assessment in critically ill patients. Iran J Med Sci, 41(3): 171-179.

Kampa, J. C. C., Reis, L. O., Mezzomo, T. R. \& Camargo, C. Q. (2020). Pacientes sob terapia nutricional enteral e prevalência de interações fármaco-nutrientes no ambiente hospitalar. Res Soc Dev, 9(3):e 62932680.

Kim, H., Stotts, N. A., Froelicher, E. S., Engler, M. M. \& Porter, C. (2013). Enteral nutritional intake in adult Korean intensive care patients. Am J Crit Care, 22(2):126-135.

Kimiaei-Asadi, H. \& Tavakolitalab, A. (2017). The assessment of the malnutrition in traumatic ICU patients in Iran. Electron Physician, 9(6): 4689-4693.

Lec, C. C. H., Yandell, R., Fraser, R. J. L., Chua, A. P., Chong, M. F. F. \& Miller, M. (2017). Association between malnutrition and clinical outcomes in the intensive care unit: a systematic review. J Parenter Enteral Nutr, 41(5): 744-758.

Lesniovski, A. P. S., Claudino, L. M., Sbalqueiro, M. E. P. \& Mezzomo, T. R. (2018). Indicadores de terapia nutricional em uma unidade de terapia intensiva de trauma em Curitiba, PR, Brasil. Nutr Clin Diet Hosp, 38(1): 149-155.

Mcclave, S. A., Taylor, E., Martindale, R. G., Warren, M. M., Johnson, D. R., Braunschweig, C., McCarthy, M. S., Davanos, E., Rice, T. W., Cresci, G. A., Gervasio, J. M., Sacks, G. S., Roberts, P. R., Compher, C, Society of Critical Care Medicine \& American Society for Parenteral and Enteral Nutrition. (2016). Guidelines for the provision and assessment of 
nutrition support therapy in the adult critically ill patient: Societyof Critical Care Medicine (SCCM) and American Society for Parenteral and Enteral Nutrition (A.S.P.E.N.). J Parenter Enteral Nutr, 40(2): 159-2011.

Menezes, N. N. B., Silva, J. T., Cutunda, L. B., de Gois, F. N. \& Oliveira, C. C. (2018). Adequação entre a terapia nutricional enteral prescrita e a dieta administrada em pacientes críticos. Nutr Clin Diet Hosp, 38(4):57-64

Oliveira Filho, R. S., Ribeiro, L. M., Caruso, L., Lima, P. A., Damasceno, N. R. \& Soriano, F. G. (2016). Quality indicators for enteral and parenteral nutrition therapy: application in critically ill pacient "at nutricional risck". Nutr Hosp, 33(5): 1027-1035.

Paese, M. C. S., Nascimento, D. B. D. \& Nascimento, J. E. A. (2016). Critical energy deficit and mortality in critically ill patients. Nutrición Hospitalaria. Nutr Hosp, 33: 552-527.

Pasinato, V. F., Barbigier, M. C., Rubin, B. A., Castro, K., Morais, R. B. \& Perry, I. D. S. (2013). Terapia nutricional enteral em pacientes sépticos na unidade de terapia intensiva: adequação às diretrizes nutricionais para pacientes críticos. Rev Bras Ter Intensiva, 25(1): 1724.

Pereira, A.S., Shitsuka, D. M., Parreira, F. J. \& Shitsuka, R. (2018). Metodologia da pesquisa científica. [e-book]. Santa Maria: Ed. UAB/NTE/UFSM. Disponível em: https://repositorio.ufsm.br/bitstream/handle/1/15824/Lic_Computacao_MetodologiaPesquisa-Cientifica.pdf?sequence=1. Acesso em: 15 Abril 2020.

Ribeiro, L. M. K., Oliveira, R. S. O., Caruso, L., Lima, P. A., Damasceno, N. R. T. \& Soriano, F. G. (2014). Adequação dos balanços energético e proteico na nutrição por via enteral em terapia intensiva: quais são os fatores limitantes? Rev Bras Ter Intensiva, 26(2): 155-162.

Rodrigues, J. B., Fraga, L., Iza, M., Melo, F. S. \& Ferreira, D. S. S. A. (2018). Fatores associados à diarreia em uma unidade de terapia intensiva: estudo de coorte. Nutr clín diet hosp, 30(2): 127-132. 
Sampaio, I. R., Ferrari, T. K. V., Toso, T. P., Duarte, L. M., Luzzi, L. C., Souza, V. V. \& Mezzomo, T. R. (2019). Análise da adequação dos indicadores de qualidade em terapia nutricional enteral em uma unidade de terapia intensiva. Res Soc Dev, 8(12):e 468121941.

Santana, M. M. A., Vieira, L. L., Dias, D. A. M., Braga, C. C. \& Costa, R. M. (2016). Inadequação calórica e proteica e fatores associados em pacientes graves. Rev Nutr, 29(5):645-654.

Souza, M. A., \& Mezzomo, T. R. (2016). Estado nutricional e indicadores de qualidade em terapia nutricional de idosos sépticos internados em uma unidade de terapia intensiva. Rev Bras Nutr Clin; 31(1): 23-8.

Tirlapur, N., Puthucheary, Z. A., Cooper, J. A., Sanders, J., Coen, P. G., Moonesinghe, S. R., Wilson, A. P., Mythen, M. G. \& Montgomery, H. E. (2016). Diarrhoea in the critically ill is common, associated with poor outcome, and rarely due to Clostridium difficile. Sci Rep, 6(24691): 1-9

Waitzberg DL. (2010). Indicadores de qualidade em terapia nutricional: aplicação e resultados. São Paulo: Institute.

\title{
Porcentagem de contribuição de cada autor no manuscrito
}

\author{
Jessica Brasil - 21,34\% \\ Larissa Marques Pereira - 21,33\% \\ Mary Evelyn Sbalqueiro Pistori - 18\% \\ Thais Regina Mezzomo - 18\% \\ Marília Rizzon Zaparolli - 21,33\%
}

